\title{
On thermal effects in a special class of viscoelastic fluids
}

By M. J. Crochet and P. M. Naghdi (Berkeley, Calif.)

Rheol. Acta 12, 237-245 (1973).

p. 237, line 5 of 2nd paragraph of section 1: Replace Colemans with Coleman's

p. 238, line 2 after eq. [2.87: Replace the number 1 with 1 .

p. 238, the second of eqs. [2.10]: Replace $T$ and $\mathscr{T}$ with $T$ and $\mathscr{T}$, respectively.

p. 238, line 1 after eq. [2.11]: Replace $\mathscr{T}$ with $\mathscr{T}$.

p. 238, line 2 after eq. [2.11]: Replace $S$ with $\mathscr{S}$.

p. 239, line 5 after eq. [2.19]: Insert 3 after "functional".

p. 239, Eq. [2.21]: The upper limit of the integral should be $\xi_{s}$.

p. 240, line 2 after eq. [3.12]: Insert the sentence "It is clear that under the constant history [2.14], in view of [3.10], the functional $\mathfrak{F}^{\prime}$ in [3.8] reduces to a constant plus the functional $\mathfrak{F}^{*}$ in [3.12]". p. 241, 2 lines above eq. [3.19]: Replace "specifid" with "specific".

p. 241, 2 lines above eq. [3.21]: Replace "nonisothermal" with "non-isothermal".

p. 241, the second of eq. [3.23]: Replace $\mathscr{T}^{*}$ with $\mathscr{T}^{*}$.

p. 241, 1 line after [3.23]: Replace the first $\mathscr{T}^{*}$ on this line by $\grave{T}^{*}$.

p. 242, the third of eqs. [4.13]: Replace $\mathfrak{s}^{*}$ with $\mathfrak{s}_{2}^{*}$.

p. 243, just above eq. [4.19]: Insert footnote 7) after "by" and add at the bottom of the page "7) see eq. [108.22] in Truesdell and Noll (6, p. 437)".

p. 243, Eq. [5.6]: Replace $T$ with $T$.

p. 243, lines $4-5$ after eq. [5.6]: Replace "velocities" by "velocity".

p. 245, the volume and page numbers and the date for reference 4) should read: 10, 775 (1972).

\section{Thermal stress analysis of glass with temperature dependent coefficient of expansion}

\author{
By S. M. Ohlberg and T. C. Woo (Harmarville \& Pittsburgh, Penna.)
}

Rheol. Acta 12, 261-264 (1973)

p. 262, Eq. [5]

read correctly: $\quad \theta(\varkappa, t)=\frac{1}{\alpha_{B}} \int_{r}^{T(x, t)} \alpha\left(T^{\prime}\right) d T^{\prime}$

\section{Rheology on the drawing zone in glass spinning}

By G. Manfrè (Novara)

Rheol. Acta 12, 265-272 (1973)

p. 265, List of symbols,

read correctly:

$T_{a} T_{s}$ Temperature of fibre at the centre and the surface $\left({ }^{\circ} \mathrm{C}\right)$

$x \quad$ Axial distance of the fibre from the nozzle exit $(\mathrm{cm})$. p. 270, right column, first eq. read correctly:

$R_{e}=\frac{\varrho R_{0} U_{0}}{\eta}$

p. 272, new address of the author:

Dr. G. Manfrè, Montecatini Edison SpA - DIPE

Centro Ricerche-Castellanza (VA), Italy. 